\title{
Noise properties of a resonance-type spin-torque microwave detector
}

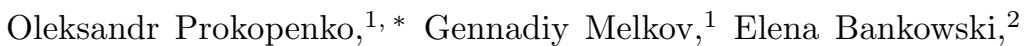 \\ Thomas Meitzler, ${ }^{2}$ Vasil Tiberkevich, ${ }^{3}$ and Andrei Slavin ${ }^{3}$ \\ ${ }^{1}$ Faculty of Radiophysics, Taras Shevchenko National University of Kyiv, Kyiv 01601, Ukraine \\ ${ }^{2}$ U.S. Army TARDEC, Warren, MI 48397, USA \\ ${ }^{3}$ Department of Physics, Oakland University, Rochester, MI 48309, USA
}

(Dated: August 19, 2018)

\begin{abstract}
We analyze performance of a resonance-type spin-torque microwave detector (STMD) in the presence of noise and reveal two distinct regimes of STMD operation. In the first (high-frequency) regime the minimum detectable microwave power $P_{\min }$ is limited by the low-frequency JohnsonNyquist noise and the signal-to-noise ratio (SNR) of STMD is proportional to the input microwave power $P_{\mathrm{RF}}$. In the second (low-frequency) regime $P_{\min }$ is limited by the magnetic noise, and the $\mathrm{SNR}$ is proportional to $\sqrt{P_{\mathrm{RF}}}$. The developed formalism can be used for the optimization of the practical noise-handling parameters of a STMD.

PACS numbers: 85.75.-d, 07.57.Kp, 84.40.-x
\end{abstract}

It has been shown in [1, 2] that a magnetic tunnel junction (MTJ) subjected to the external microwave current $I_{\mathrm{RF}}(t)=I_{\mathrm{RF}} \cos (2 \pi f t)$ can perform as a resonance-type quadratic detector of microwave radiation generating the DC voltage $U_{\mathrm{DC}}$ proportional to the acting microwave power $U_{\mathrm{DC}}=\varepsilon P_{\mathrm{RF}}\left(P_{\mathrm{RF}} \sim I_{\mathrm{RF}}^{2}\right)$. The detector operation is based on the spin-torque effect [3, 4] and the detector sensitivity $\varepsilon$ has a maximum value $\varepsilon=\varepsilon_{\text {res }}$ when the frequency of the external microwave signal is close to the eigen-frequency $f_{0}$ of the MTJ nanopillar, $f=f_{0}$.

The resonance sensitivity $\varepsilon_{\text {res }}$ of the spin-torque microwave detector (STMD) was calculated in [5]:

$$
\varepsilon_{\mathrm{res}}=\frac{U_{\mathrm{DC}}}{P_{\mathrm{RF}}}=\left(\frac{\gamma \hbar}{4 e}\right) \frac{P^{3}}{M_{s} V \Gamma} Q\left(\theta_{0}\right),
$$

where $\gamma \approx 2 \pi \cdot 28 \mathrm{GHz} / \mathrm{T}$ is the modulus of the gyromagnetic ratio, $\hbar$ is the reduced Planck constant, $e$ is the modulus of the electron charge, $P$ is the spin-polarization efficiency of the MTJ, $M_{s}$ is the saturation magnetization of the free layer (FL) of MTJ, $V=\pi r^{2} d$ is the volume of the FL ( $r$ is its radius and $d$ is its thickness), $\Gamma$ is the magnetization damping rate in the MTJ FL proportional to the Gilbert damping constant $\alpha$, and $Q\left(\theta_{0}\right)$ is the geometrical factor that depends on the angle $\theta_{0}$ between the directions of the equilibrium magnetization in FL and pinned layer (PL) of the MTJ. For an in-plane magnetized MTJ, $Q\left(\theta_{0}\right)=\sin ^{2} \theta_{0} /\left(1+P^{2} \cos \theta_{0}\right)^{2}$.

Estimations based on Eq. (11) 5] and recent experimental results [2, 6] have demonstrated that the STMD sensitivity can exceed that of passive semiconductor Schottkydiode microwave detectors $(\varepsilon \sim 1000 \mathrm{~V} / \mathrm{W})$, which makes STMD very interesting for practical applications in microwave measurement technology.

The operation and the minimum detectable power of all types of microwave detectors are limited by noise

*Electronic address: ovp@univ.kiev.ua (in particular, by the low-frequency Johnson-Nyquist (JN) noise in the case of unbiased Schottky diodes [7]), and, therefore, it is important to understand the noisehandling properties of the novel STMD based on MTJ.

In this Letter we present theoretical analysis of the noise properties of a passive STMD (no DC bias current) using the STMD model developed in [5] with additional terms describing influence of thermal fluctuations. In our analysis we took into account three sources of noise:

(a) Low-frequency Johnson-Nyquist (JN) noise voltage $U_{\mathrm{N}}(t)$ associated with the MTJ resistance $R_{0}$. This type of noise is additive and is independent of the magnetization dynamics.

(b) High-frequency JN noise current $I_{\mathrm{N}}(t)$ which transforms into a non-additive low-frequency noise after mixing with the microwave oscillations caused by the input microwave signal.

(c) Magnetic noise (MN), which is caused by the thermal fluctuations of the magnetization direction in the MTJ FL. This noise, modeled by a random magnetic field $\boldsymbol{B}_{\mathrm{N}}(t)$, leads to the fluctuations of the electrical resistance of the STMD and transforms to low frequencies after mixing with the driving current $I_{\mathrm{RF}}(t)$.

The dynamics of magnetization $\boldsymbol{M}$ in the MTJ FL under the action of a microwave current containing both deterministic and noise components $I(t)=I_{\mathrm{RF}}(t)+I_{\mathrm{N}}(t)$ and noise magnetic field $\boldsymbol{B}_{\mathrm{N}}(t)$ is described by the Landau-Lifshits-Gilbert-Slonczewski equation:

$$
\begin{aligned}
\frac{d \boldsymbol{M}}{d t}= & \gamma\left[\boldsymbol{B}_{\mathrm{eff}}(\boldsymbol{M}) \times \boldsymbol{M}\right]+\frac{\alpha}{M_{s}}\left[\boldsymbol{M} \times \frac{d \boldsymbol{M}}{d t}\right]+ \\
& \frac{\sigma I(t)}{M_{s}}[\boldsymbol{M} \times[\boldsymbol{M} \times \boldsymbol{p}]]+\gamma\left[\boldsymbol{B}_{\mathrm{N}}(t) \times \boldsymbol{M}\right],
\end{aligned}
$$

where $\boldsymbol{B}_{\text {eff }}(\boldsymbol{M})$ is the effective magnetic field, which includes the external bias magnetic field $\boldsymbol{B}_{0}$ and the demagnetization field, $\boldsymbol{p}$ is the unit vector in the direction of the magnetization of the MTJ PL, $\sigma=(\gamma \hbar / 2 e) P /[(1+$ $\left.\left.P^{2} \cos \theta\right) M_{s} V\right]$ is the current-torque proportionality coefficient, and $\theta$ is the angle between vectors $\boldsymbol{M}$ and $\boldsymbol{p}$. 
We performed analysis for a "planar" STMD, in which both FL and PL are magnetized in-plane. In this case, the MTJ eigen-frequency is $f_{0}=$ $(\gamma / 2 \pi) \sqrt{B_{0}\left(B_{0}+\mu_{0} M_{s}\right)}$ and the damping rate has the form $\Gamma=\alpha \gamma\left(B_{0}+\mu_{0} M_{s} / 2\right)$, where $\mu_{0}$ is the vacuum susceptibility. We assumed that $f=f_{0} \gg \Gamma /(2 \pi)$. Using Eq. (2), we found the linear FL magnetization response to current $I(t)$ and field $B_{\mathrm{N}}(t)$ in the frequency domain. The output STMD signal was calculated as the lowfrequency part of the voltage $\left[R(\theta) I(t)+U_{\mathrm{N}}(t)\right]$, where $R(\theta)=R_{\perp} /\left(1+P^{2} \cos \theta\right)$ is the MTJ magnetoresistance, $R_{\perp}=\mathrm{RA} /\left(\pi r^{2}\right)$ is the MTJ resistance in the perpendicular magnetic state $(\theta=\pi / 2)$, and $\mathrm{RA}$ is the resistancearea product of the MTJ. We assumed that all noise sources are independent Gaussian processes with uniform spectral densities $S\left(U_{\mathrm{N}}\right)=S\left(I_{\mathrm{N}}\right) R_{0}^{2}=2 k_{B} T R_{0}$ and $S\left(B_{\mathrm{N}}\right)=2 \alpha k_{B} T /\left(\gamma M_{s} V\right)$, where $k_{B}$ is the Boltzmann constant, $T$ is the noise temperature, and $R_{0}=R\left(\theta_{0}\right)$ is the equilibrium resistance of MTJ. The detailed derivation of the noise spectrum of STMD will be presented elsewhere [8].

We found that the noise of the output voltage $U_{\mathrm{DC}}=$ $\varepsilon_{\text {res }} P_{\mathrm{RF}}$ has characteristic spectral width of $\Gamma$ and, for typical frequency bandwidth of measurement $\Delta f \ll$ $\Gamma /(2 \pi)$, can be considered as frequency-independent. The root mean square fluctuations $\Delta U_{\mathrm{DC}}$ can be written in the simple form

$$
\Delta U_{\mathrm{DC}}=U_{\mathrm{JN}} \sqrt{1+\frac{U_{\mathrm{DC}}}{U_{\mathrm{IM}}}+\frac{U_{\mathrm{DC}}}{U_{\mathrm{MN}}}},
$$

where the three terms in the right-hand side part of the equation describe, respectively, the influence of the three above mentioned noise sources and

$$
\begin{aligned}
U_{\mathrm{JN}} & =\sqrt{4 k_{B} T R_{0} \Delta f}, \\
U_{\mathrm{IM}} & =\frac{R_{0}}{4 \varepsilon_{\mathrm{res}}}, U_{\mathrm{MN}}=\frac{\gamma \hbar}{2 e} \frac{B_{0}}{P} .
\end{aligned}
$$

For typical parameters (see e.g. [5, 9]) of an MTJ nanopillar $\left(r=50 \mathrm{~nm}, d=1 \mathrm{~nm}, P=0.7, \theta_{0}=45 \mathrm{deg}\right.$, $R_{0}=500 \Omega$ (giving $\mathrm{RA}=5.29 \Omega \mu \mathrm{m}^{2}$ ), $\alpha=0.01$, $\mu_{0} M_{s}=0.8 \mathrm{~T}, B_{0}=38 \mathrm{mT}$ (giving $f_{0}=5 \mathrm{GHz}$ ) Eq. (11) gives the resonance STMD sensitivity in the passive regime $\varepsilon_{\text {res }} \approx 700 \mathrm{~V} / \mathrm{W}$, which is comparable to the sensitivity of Schottky diodes 9]. At room temperature $T=300 \mathrm{~K}$ and for the measurement bandwidth of $\Delta f=1 \mathrm{MHz}$ Eqs. (44) give the following estimations for the noise-induced voltages: $U_{\mathrm{JN}}=2.88 \mu \mathrm{V}$, $U_{\mathrm{IM}}=175.9 \mathrm{mV}, U_{\mathrm{MN}}=3.14 \mu \mathrm{V}$.

It is clear that the characteristic voltage $U_{\text {IM }}$ of the non-additive high frequency JN noise is much larger than the voltages created by the low frequency $\mathrm{JN}$ noise $U_{\mathrm{JN}}$, magnetic noise $U_{\mathrm{MN}}$, and the typical DC voltage output $U_{\mathrm{DC}}$ of the STMD. This means that the influence of the high-frequency JN noise in Eq. (3) can be completely ignored. Note, also, that, in contrast with the other characteristic noise voltages, the voltage $U_{\mathrm{MN}}$ caused by the magnetic noise is proportional to the bias magnetic field

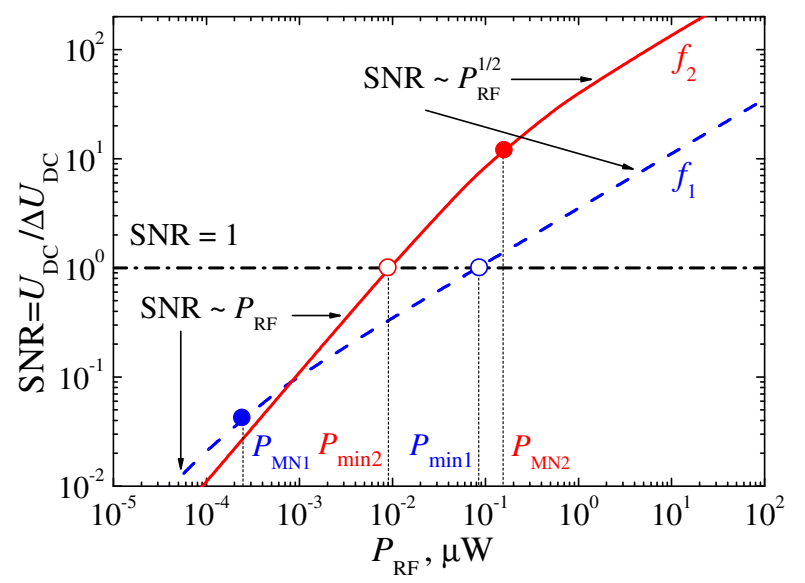

FIG. 1: Dependence of the SNR of STMD on the input microwave power $P_{\mathrm{RF}}$ calculated from Eq. (5) for two different frequencies of the input microwave signal: $f_{1}=1 \mathrm{GHz}$ (dashed blue line) and $f_{2}=25 \mathrm{GHz}$ (solid red line). All other parameters are the same as indicated below Eqs. (41). $P_{\min }$ is the minimum detectable power of $\operatorname{STMD}($ at $\operatorname{SNR}=1$ ) and $P_{\mathrm{MN}}$ is the frequency-dependent characteristic power of magnetic noise.

$B_{0}$, and, therefore, increases with the increase of the frequency of the input microwave signal.

Now, introducing the microwave powers $P_{\mathrm{RF}}=$ $U_{\mathrm{DC}} / \varepsilon_{\mathrm{res}}, P_{\mathrm{JN}}=U_{\mathrm{JN}} / \varepsilon_{\mathrm{res}}, P_{\mathrm{MN}}=U_{\mathrm{MN}} / \varepsilon_{\text {res }}$ and using Eq. (3), we can write a simple expression for the signalto-noise ratio (SNR) of the STMD in terms of these characteristic powers:

$$
\mathrm{SNR}=\frac{U_{\mathrm{DC}}}{\Delta U_{\mathrm{DC}}}=\frac{P_{\mathrm{RF}}}{P_{\mathrm{JN}}} \sqrt{\frac{P_{\mathrm{MN}}}{P_{\mathrm{MN}}+P_{\mathrm{RF}}}} .
$$

The simple analysis of Eq. (5) demonstrates that there are two distinct regimes of operation of the resonance STMD in the presence of thermal noise. We shall classify them by the type of noise that limits the minimum detectable power of STMD $P_{\min }$ (power corresponding to $\mathrm{SNR}=1)$.

The first regime corresponds to the case of relatively high frequencies of the input microwave signal, when $P_{\mathrm{MN}} \gg P_{\mathrm{RF}}\left(\right.$ for $\left.P_{\mathrm{RF}} \sim P_{\mathrm{min}}\right)$. In this regime, similar to the conventional semiconductor diodes, the minimum detectable power is limited by the low-frequency JN noise, $P_{\min }=P_{\mathrm{JN}}$, and the SNR of STMD is linearly proportional to the input microwave power $P_{\mathrm{RF}}$ $\left(\mathrm{SNR} \simeq P_{\mathrm{RF}} / P_{\mathrm{JN}}\right)$.

The second regime takes place in the opposite limiting case of relatively low input frequencies, when $P_{\mathrm{MN}} \ll$ $P_{\mathrm{RF}}$. In this case the SNR of STMD increases with $P_{\mathrm{RF}}$ much slower than in conventional diodes, and is proportional to the square root of the input microwave power: $\mathrm{SNR} \simeq \sqrt{P_{\mathrm{RF}} / P_{\min }}$. The minimum detectable power $P_{\mathrm{min}}=P_{\mathrm{JN}}^{2} / P_{\mathrm{MN}}$ in this regime is limited by the magnetic noise in the FL of the MTJ.

We note, that if at a fixed frequency of the input signal the SNR of an STMD is measured in a wide range of 


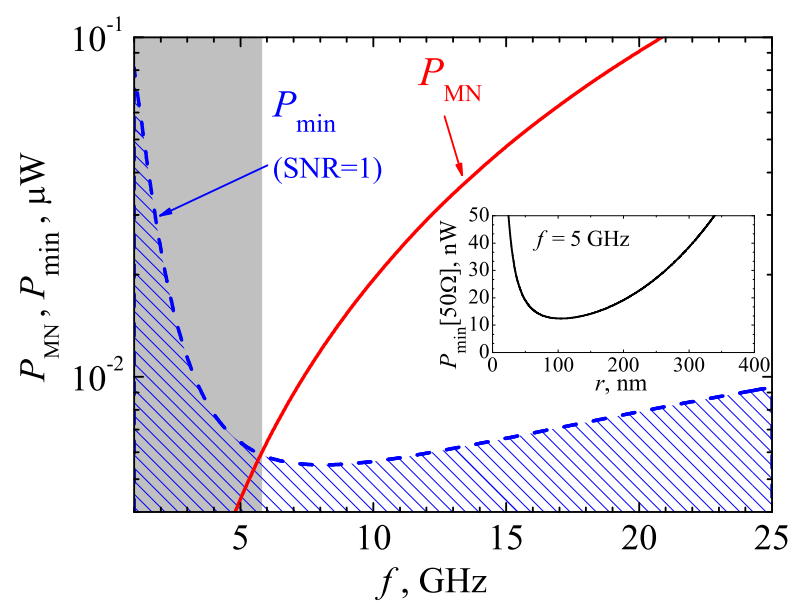

FIG. 2: Characteristic power of magnetic noise $P_{\mathrm{MN}}$ (solid red line) and minimum detectable power $P_{\min }$ of STMD (dashed blue line) as functions of the input microwave frequency $f$. The blue dashed area corresponds to undetectable signals $P_{\mathrm{RF}}<P_{\min }$ and gray shaded area shows the lowfrequency STMD regime, where the magnetic noise is dominant in the whole practical region $P_{\mathrm{RF}}>P_{\min }$. Inset: minimum detectible microwave power delivered to a $50-\Omega$ transmission line $P_{\min }[50 \Omega]$ for $f=5 \mathrm{GHz}$ as a function of the radius $r$ of the MTJ nanopillar. STMD parameters are indicated below Eqs. (4).

input powers, covering both the above described limiting cases, it is possible to find the characteristic power of the magnetic noise $P_{\mathrm{MN}}$ with a good accuracy, and, using expression (4b) for $U_{\mathrm{MN}}$, to determine with the same accuracy the MTJ spin-polarization efficiency $P$.

The existence of two distinct regimes of STMD operation is illustrated in Fig. 1, where two curves calculated from Eq. (5) show the STMD SNR as functions of the input power for signal frequencies $f_{1}=1 \mathrm{GHz}$ (dashed blue line) and $f_{2}=25 \mathrm{GHz}$ (red solid curve). It can be seen, that both curves (presented in logarithmic coordinates) demonstrate the clear change of slope from 1 to $1 / 2$ in the region, where the input power $P_{\mathrm{RF}}$ is close to the characteristic power of the magnetic noise $P_{\mathrm{MN}}$ (which increases with the increase of the input signal frequency). The minimum detectable power $P_{\min }($ corresponding to SNR $=1$ ) in the high-frequency case is smaller than $P_{\mathrm{MN}}$ and lies in the region of the linear dependence of SNR on $P_{\mathrm{RF}}$ (solid red line in Fig. 11). The situation is opposite in the low frequency case (blue dashed curve in Fig. 1), when $P_{\min }>P_{\mathrm{MN}}$ and lies in the region, where the slope of the SNR curve is equal to $1 / 2$.

The evolution of the characteristic powers $P_{\mathrm{MN}}$ and $P_{\min }$ with the increase of frequency of the input microwave signal is shown in Fig. 2, The curve $P_{\mathrm{MN}}(f)$ separates the plane into the region, where magnetic noise is dominant (above the curve), and the region, where the STMD operation is limited by the JN noise (below the curve). It is, clear, that the smallest detectable power is achieved near the border of these two regimes.

When an STMD based on an MTJ nanopillar is used as a sensor of microwave radiation, it is typically connected to a standard transmission line with the impedance of $Z_{\mathrm{TL}}=50 \Omega$. The minimum detectable microwave power delivered to a 50- $\Omega$ transmission line can be written as [10] $P_{\min }[50 \Omega]=(1 / 4)\left(R_{0}+Z_{\mathrm{TL}}\right)^{2} P_{\min } / Z_{\mathrm{TL}} R_{0}$. Using this expression and taking into account the size dependence of the STMD resistance $\left(R_{0} \propto 1 / r^{2}\right)$, it is possible to show that $P_{\min }[50 \Omega]$ has a clear minimum as a function of the nanopillar radius $r$. For instance, the optimum value of the nanopillar radius is $r_{\mathrm{opt}} \approx 100 \mathrm{~nm}$ for the input frequency $f=5 \mathrm{GHz}$ (see the inset in Fig. (2)).

In conclusion, we have demonstrated that STMD in the presence of noise can operate in two distinct regimes, one of which is limited by magnetic noise and is different from the regime of operation of traditional semiconductor detectors. We have, also, suggested that the measurements of STMD SNR in a wide range of input powers can be used to determine the spin-polarization efficiency $P$ of MTJ nanopillars, and have shown that the developed formalism can be used for the optimization of noise-handling parameters of a STMD.

This work was supported in part by the Contract from the U.S. Army TARDEC, RDECOM, by the grants ECCS-1001815 and DMR-1015175 from the National Science Foundation of the USA, by the Grant No. M/902010 from the Ministry of Education and Science of Ukraine, and by the Grant No. UU34/008 from the State Fund for Fundamental Research of Ukraine.
[1] A.A. Tulapurkar, Y. Suzuki, A. Fukushima et al., Nature (London) 438, 339 (2005).

[2] S. Ishibashi, T. Seki, T. Nozaki et al., Abstracts of the IEEE 7th International Symposium on Metallic Multilayers (MML 2010), I-40, Berkeley, California, September 2010.

[3] J.C. Slonczewski, J. Magn. Magn. Mat. 159, L1 (1996).

[4] L. Berger, Phys. Rev. B 54, 9353 (1996).

[5] C. Wang, Y.-T. Cui, J.Z. Sun et al., J. Appl. Phys. 106, 053905 (2009).

[6] X. Cheng, C.T. Boone, J. Zhu, and I.N. Krivorotov,
Phys. Rev. Lett. 105, 047202 (2010).

[7] N.B. Lukyanchikova, Noise research in semiconductor physics, (CRC Press, Amsterdam, 1996).

[8] O. Prokopenko, V. Tiberkevich, and A. Slavin, in preparation.

[9] S. Ishibashi, T. Seki, T. Nozaki et al., Appl. Phys. Express 3, 073001 (2010).

[10] D.M. Pozar, Microwave Engineering, 3rd ed. (Wiley, New York, 2005). 